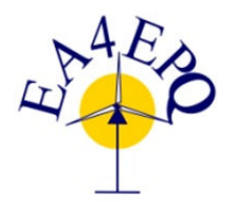

$18^{\text {th }}$ International Conference on Renewable Energies and Power Quality (ICREPQ'20)

Granada (Spain), $1^{\text {st }}$ to $2^{\text {nd }}$ April 2020

Renewable Eenergy and Pourer Quality fournal (RE\&PQJ)

ISSN 2172-038 X, Volume No.18, June 2020

\title{
Optimal Allocation of Energy Storage Systems for Load Management in Distributed Renewable Generations
}

\author{
Y.M.Al-Humaid ${ }^{1}$, M.A.Abdulgalil ${ }^{1}$, K.A.Khan ${ }^{1}$, and M.Khalid ${ }^{1,2}$ \\ ${ }^{1}$ Department of Electrical Engineering \\ King Fahd University of Petroleum and Minerals (KFUPM) \\ Dhahran 31261, Kingdom of Saudi Arabia \\ Email: g201702290@kfupm.edu.sa, atta@kfupm.edu.sa, g201604320@kfupm.edu.sa \\ ${ }^{2}$ King Abdullah City for Atomic and Renewable Energy (K. A. CARE), Energy Research \& Innovation Center \\ Dhahran 31261, Kingdom of Saudi Arabia \\ Email: mkhalid@kfupm.edu.sa
}

\begin{abstract}
In recent years, the concept of smart grids has brought major development in distributed systems. Therefore, the overall grid efficiency can be enhanced with intended integration of several different types of renewable energy sources in distribution systems and further target highest reliability and reduced system losses with great operational flexibility. In this paper, we address optimal allocation of storage devices for achieving proper and feasible satisfactory load management. The allocation strategy consists of AC- optimal power flow (AC-OPF) for suitable storage placement with the objective to optimize the installation cost, energy balance and system losses. The proposed methodology is tested for various scenarios of renewable integrated, IEEE 24-bus standard test network. Hence, the overall system benefits is observed to be maximized.

Index Terms-AC-OPF, cost optimization, demand side management, energy storage system, smart grid.
\end{abstract}

\section{INTRODUCTION}

The concept of Smart grids are widely implemented in utility power industry for benefits in environmental as well as economical conditions and this was enabled by evolving to distribution network, which allows small generators to be connected in the system. Many governments are planning to increase the use of renewable resources into local along with regional grids to meet the highest possibility for emission reduction. In addition, the system will have independence energy sources which hence provides system reliability. One of the most widely used facilitation for penetration increment of renewable resources along with diesel generators (DG) is the integration of energy storage systems (ESS). One of the beneficial features of ESS is the shaving of the peak load which implies power exchange between ESS and generation units. Therefore, storing of power during off peak periods and during peak periods the stored power can be discharged or sold to utility grid. This perspective of ESS implementation is economical for ESS owners, as well as, power system planners for planning ahead reserve energy storage with consideration of power prediction of wind energy depending on accuracy reading of forecast [1]-[5].

In this front, several propositions has been presented over the years. The study in [6] presents a techniques for reduction of forecast errors. In [7], using of non dominated storing genetic algorithm optimization technique this study presents the best practical approaches for load management by evaluating energy storage impact on net present value (NPV) on specified costs. Similarly, in [8], the optimization for energy storage use and furhter reduction of fuel consumption by the proposed methodology of utilizing stochastic optimization for storage sizing which consequently result in major financial compensation for operation as well as reduction of fuel costs.

Accordingly, many papers have addressed the challenges of sizing ESS for reducing uncertainty of short term of wind power [9]. Impressive probabilistic method is proposed in [10], shows the spectral analysis of solar resources and wind energy linked with daily load profiles. This method is applied on an off grid system where the calculation of storage is formulated for different levels of mean load. Furthermore, other study presents worst case assumptions, on the basis formulates the acquisition of the unsaved energy and hence recommend an optimal ESS size, [10]. An interesting approach of identifying 
the optimal energy storage operation during specified period based on the optimization of ESS sizing, and forecasting the stochastic nature of system by applying time series model. Although there are complication of forecasting wind energy and solar radiation output. However, the method of time series provides an optimal solution. The uncertainties in PV and wind power leads to errors, which inherently induces voltage fluctuations, so the best power forecasting method is used to predict the renewable energy output and power demand to obtain the best determination of energy storage capacity with accurate results [11]. The authors in [12], studied sizing battery storage by probabilistic method to manage and mitigate the uncertainty in the net load linked with stand-alone power plant. Similar condition is proposed in [13], in this study, a control strategy for co-optimization and sizing for an existing PV power plant is implemented and proposed for the optimization of global linear programming (LP) algorithm, where the same optimization as operating management of ESS is computed for the optimal components sizing. In [14], authors proposed a district energy system (DES) with thermal energy and power generation along with grid connected system, the study was applied on Monto carlo to analyse stochastic power generation from renewable energy resources in DES to aim mitigating the operating costs. The basic contribution of this paper can be briefly summarized as following:

- The paper presents a short-term planning framework considering 24 hours, the objective of maximization of profits is fulfilled in the various scenarios of the test distribution network associated with optimal distributed system (DS) sizing and ESS allocation.

- Implementation of time series pattern to optimize the the size of DS, and further consider different ESS, with 16 cases to meet the system demand in the worst case scenario.

- Further, the DS units is maintained under optimized operation in each state of the load demand.

\section{Problem Formulation}

The optimal power flow (AC-OPF) is the optimal operational schedule that consists of determining on how many generators and storage units is required to meet the entire load demand while satisfying the physical bounds and to optimize the cost function on storage system, power exchanged with network of utility grid and generators. Therefore, the objective of this paper is to optimally size ESS and to solve OPF problem in power system. OPF objective is to calculate the power flow through transmission lines and the results are subjected to the constraints power flow limits in the transmission lines. moreover, OPF is used to compute the optimal selection of each generator. The OPF can be DC-OPF or AC-OPF and the approach in this paper is AC-OPF with objective to optimize the total cost includes with operational and investment of storage systems, operating and maintenance costs which is formulated in the following equation.

$$
\operatorname{minCM} G_{\text {units }}+C M G_{\text {ex }}+I C_{E S S}
$$

where $C M G_{\text {units }}$ is the cost of operation for distributed generators of microgrid, $C M G_{e x}$ is the revenue cost for the imported power which is exchanged from the main utility grid or in other way exported to the grid, where $I C_{E S S}$ is cost for capital investment required to establish the storage system. For the calculation of operation cost of distributed generators for microgrid is formulated in (2). the variables $z, y$ and $u$ are binary. Which means they are either 0 or 1 . which indicates if $u_{i, t}$ is 0 that means generator $i$ at time $t$ is in OFF position while if $u_{i, t}$ is 1 that means the generator $i$ at time $t$ in ON state. In addition to that if $y_{i, t}$ that indicates the start up of generator $i$ at hour $t$. Moreover, $z_{i, t}$ the generator $i$ is shutdown at hour $t$. So, the $z_{i, t}$ and $y_{i, t}$ both are 1 during the first hour of startup of the generator and shutdown respectively. where values of $z_{i, t}$ and $y_{i, t}$ both are 0 at the rest of the time. since the values of $z_{i, t}, y_{i, t}$ and $u_{i, t}$ are integers, the mixed integer linear programming (MILP) has to be used for problem optimization. whereas the fixed cost of units F, and i is fixed when the unit $i$ is in ON state. This cost is computed in all hours in the unit is obliged at. However, the unit power output is not linked with calculating the fixed cost, where the cost variable of units $V$ and $i$ is not fixed (variable) and it depends on the unit $i$ of the power output. $z_{i, t}, y_{i, t}$ and $u_{i, t}$ all are binary variables indicate the obligation state of unit $i$ at time $t$, shutdown signal of unit $t$ at time $t$ and startup signal of unit $i$ at time $t$.

$$
\left.\left.C M G_{\text {units }}=\sum_{t=1}^{N T} \sum_{i=1}^{N I}\right] F_{i} u_{i, t}+V_{i} P_{i, t}+S U_{i y i, t}+S D_{i z, t}\right]
$$

where $i$ indicates for the unit index and NI is the unit number, $t$ indicates for the hour index, NT is the number of hours, $F_{i}$ is no load cost for the unit $i, V_{i}$ is the not fixed cost (variable) for unit $i$ and related to power ouput of unit $i, P_{i, t}$ is power output of unit $i$ at time $t, S D_{i}$ it is the cost of shutdown for unit $i, S U_{i}$ is the cost of unit $i$ in startup. the cost function of generator cost is quadratic which is nonlinear.however, in equation (2), it was linearized to have faster and simpler optimization model. Quadratic function approach can be used for accurate results. In equation (3) shows how to calculate the cost of exported and imported power from or to the main grid. The objective function is the cost so, when the power is imported from the grid the cost will be positive. However, when the power is exported to the grid the cost will be negative.

$$
C M G_{e x}=\sum_{t=1}^{N T} \gamma P_{L_{t}}
$$

where $\gamma$ is the price of electricity per one megawatt of power sold to the grid or bought from the grid and $P_{L_{t}}$ is the power exchange from the grid or to the grid where it could be positive when the power flow is going from the grid to microgrid or negative when the power flow from microgrid to the main grid. Investment cost of ESS formulated in equation (4). The parameters are the unit prices of ESS energy and 
power. Moreover, the desicion variables are energy and rated power of ESS which are two variables representing the optimal size of ESS.

$$
I C_{E S S}=P C_{E S S} P_{E S S}^{R}+E C_{E S S} E_{E S S}^{R}
$$

Where $P C_{E S S}$ the cost of ESS power in one megawatt and $P_{E S S}^{R}$ is the rated power of storage energy, $E C_{E S S}$ is cost of the ESS energy in one megawatt hour and $E_{E S S}^{R}$ is rated energy for the storage system.

\section{SySTEM CONSTRAINTS}

System constraints includes the power constraint of generator along with renewable resources output must be equal to the load demand and if the generation is more than demand or less it will effect the system frequency and consequently the system will be unbalance, in some cases the emissions constrains shall be taken into account in some optimization problems. The objective might be minimizes the emission and the total cost in the same time in multi objective unit commitment problems [15]. In this paper the demand balance constraint is formulated as:

$$
\sum_{i=1}^{N I} P_{i, t}+P_{E S S_{t}}+P_{L}-\left(P_{W_{t}}+P_{P V_{t}}\right)=L_{t}
$$

where NI is the number of units, $P_{E S S}$ power discharged or stored at hour t, $P_{W_{t}}$ is the power wind at time t, $P_{P V_{t}}$ power of solar energy at time t, $L_{t}$ load demand at time t, in storage system $P_{E S S}$ the system is having alternative way which is either to produce or to store so, the sign in this matter is convection depend on the situation. Wind, solar energy power as well as the demand load are considered fixed and it is illustrated in table I.

TABLE I. Wind, Solar and demand parameters

\begin{tabular}{|l|l|l|l|}
\hline & Wind & Solar & Demand \\
\hline$t_{1}$ & 0.078666 & 0 & 0.684511335 \\
\hline$t_{2}$ & 0.086666 & 0 & 0.64412269 \\
\hline$t_{3}$ & 0.117333 & 0 & 0.613069156 \\
\hline$t_{4}$ & 0.258666 & 0 & 0.599733 \\
\hline$t_{5}$ & 0.361333 & 0 & 0.588874071 \\
\hline$t_{6}$ & 0.56666 & 0 & 0.59801867 \\
\hline$t_{7}$ & 0.650666 & 0.0131525 & 0.626786054 \\
\hline$t_{8}$ & 0.5666 & 0.1202729 & 0.651743189 \\
\hline$t_{9}$ & 0.484 & 0.24689697 & 0.706039246 \\
\hline$t_{10}$ & 0.548 & 0.3037889 & 0.787007049 \\
\hline$t_{11}$ & 0.7573 & 0.6357696 & 0.839016956 \\
\hline$t_{12}$ & 0.7106 & 0.9069827 & 0.852733854 \\
\hline$t_{13}$ & 0.8706 & 1 & 0.870642 \\
\hline$t_{14}$ & 0.932 & 0.8854537 & 0.834254144 \\
\hline$t_{15}$ & 0.966 & 0.763103338 & 0.816536483 \\
\hline$t_{16}$ & 1 & 0.378824638 & 0.81939417 \\
\hline$t_{17}$ & 0.8693 & 0.087837357 & 0.874071252 \\
\hline$t_{18}$ & 0.6653 & 0.0000307163 & 1 \\
\hline$t_{19}$ & 0.656 & 0 & 0.983615927 \\
\hline$t_{20}$ & 0.56133 & 0 & 0.936368832 \\
\hline$t_{21}$ & 0.56533 & 0 & 0.887597638 \\
\hline$t_{22}$ & 0.556 & 0 & 0.809297009 \\
\hline$t_{23}$ & 0.724 & 0 & 0.745856354 \\
\hline$t_{24}$ & 0.84 & 0 & 0.733473042 \\
\hline & & &
\end{tabular}

power exchanged from the storage system to the grid is limited and this depends on transmission line capacity which should be negative when the power is discharged from ESS and positive when the power is imported from the grid, the constraints shall be formulated as:

$$
-P_{L}^{\max } \leq P_{L_{t}} \leq P_{L}^{\max }
$$

where $P_{L}^{\max }$ the maximum of transmission line capacity that allow power flow going to grid or imported from the main grid.

power generation is always within the operating limits according to the capacity of unit $i$ at time $t$ as in equations (7) and (8).

$$
\begin{aligned}
& P_{i, t} \geq P_{L}^{\min } u_{i, t} \\
& P_{i, t} \leq P_{L}^{\max } u_{i, t}
\end{aligned}
$$

where $P_{L}^{\min }$ states the minimum power can be achieved by unit $i$ where $P_{L}^{\max }$ states the maximum power output of unit $i$ where $u_{i, t}$ indicates the state of the unit $i$ at time $t$. Each generation unit has maximum increment and minimum in decreasing the capacity at each time $t$ which it could be defined as ramp up and ramp down, these constraint has to be met and it can be formulated as following equation

$$
P_{i, t} \leq P_{i, t-1}+R U_{i} u_{i, t}
$$

$R U_{i}$ is the ramp up rating at unit $i$

$$
P_{i, t} \geq P_{i, t-1}-R D_{i} u_{i, t}
$$

where $R D_{i}$ is the ramp down rating at unit $i$, so the unit would be remain in its state for some time before it increase or decreases to the second state according to the constraints applied

\section{Case Study}

The system used in case study is 24-bus as shown in Fig. 1. The proposed rated load profile data have been taken from IEEE 24-bus RTS [16]. A grid connected microgrid is examined to size ESS under wind and PV panels for 24 hours. The fixed and variables costs of the equipment for the system are given in Table I [17].

TABLE II. Fixed and variable costs of batteries

\begin{tabular}{|l|l|l|l|}
\hline Type of battery & $\$ / \mathrm{W}$ & $\$ / \mathrm{Wh}$ & Efficiency(\%) \\
\hline Lead-acid & 0.2 & 0.2 & $70 \%$ \\
\hline NiCd & 0.5 & 0.4 & $85 \%$ \\
\hline Li-ion & 0.9 & 0.6 & $98 \%$ \\
\hline NaS & 0.35 & 0.3 & $95 \%$ \\
\hline
\end{tabular}




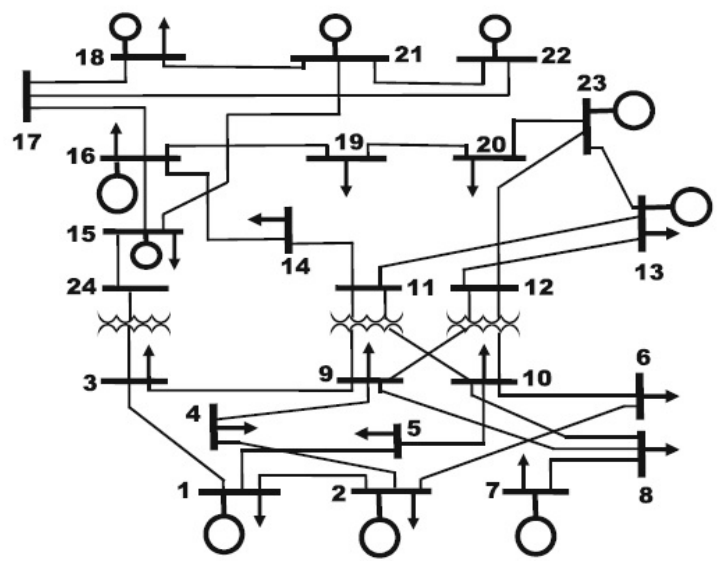

Fig. 1: IEEE 24-Bus RTS system under study

\section{RESUlts}

This section summarizes the outcomes of this study, in which bus distributed generators shall be allocated for optimal solution based on AC-OPF in 24 hours horizon to achieve load management, and thus system losses minimizes as well as cost is optimized. The approach used to analyse the allocation impact of DG types are pre-allocated through four different cases. the system without renewable energy sources, the system with wind energy only, the system with solar energy only and system with hybrid (Solar \& wind). In each case it has four different scenario, since the study was applied in four different storage systems which are (Lead Acid, NiCd, Li-ion, Nas).

\section{A. No renewable energy}

In this case, the system is assumed to be operated by DG only. As shown in Fig. 2 the optimal output power and energy for each storage system which indicated the Nas type of battery are the best selection in terms of energy and total cost required, where the highest price goes to Lead Acid type although the power \& energy were low, total cost are decreased from $\$ 0.532$ million to $\$ 0.518$ million which resulting $2.63 \%$ total savings. worth wile to mention these costs depend on the system. More savings could be achieved when DS have been taken into consideration such as improving the reliability and applying all mitigation required for rectifying all power quality issues.

\section{B. Wind only}

Only wind based is assumed in this case, the wind tubine are connected at buses 8, 19 and 21 as shown in Fig. The

TABLE III. No renewable case

\begin{tabular}{|l|l|l|l|}
\hline & $P_{E S S}(\mathrm{MW})$ & $E_{E S S}(\mathrm{MWh})$ & Total cost $(\$)$ \\
\hline Lead-acid & 251.6 & 819.5 & 542491.7 \\
\hline NiCd & 300.9 & 0.4 & 528643.2 \\
\hline Li-ion & 336.7 & 2017.4 & 525353.1 \\
\hline NaS & 499.1 & 0.3 & 518223.8 \\
\hline
\end{tabular}
table, where the total cost was the highest in Lead acid type and it the lowest was in Nas type since the price is decreased $\$ 0.414$ million to $\$ 0.407$ million which save almost $1.7 \%$ of the total price. However, using wind energy along with DG in best case scenario it could save $21.4 \%$ compared to DG's only in its best case.

TABLE IV. Wind only case

\begin{tabular}{|l|l|l|l|}
\hline & $P_{E S S}(\mathrm{MW})$ & $E_{E S S}(\mathrm{MWh})$ & Total cost(\$) \\
\hline Lead-acid & 278.6 & 1076.2 & 414760.5 \\
\hline NiCd & 277.5 & 1276.2 & 413823.5 \\
\hline Li-ion & 255 & 1034.8 & 413800.2 \\
\hline NaS & 342.8 & 1869.5 & 407846.7 \\
\hline
\end{tabular}

\section{Solar only}

Only solar based is assumed, PV cells are at buses 3, 10, and 14. as shown in Table the optimal energy and power output for each ESS, it indicates the Nas type are the optimum, since it saves from the total cost required for lead acid case which is $\$ 0.514$ to $\$ 0.500$ so, the savings almost $3 \%$ from the total cost. However, solar energy is producing more power output than the wind turbine case but with higher cost. More energy storage systems are needed to reduce the cost.

\section{Wind and solar}

In this case the assumptions were to have Hybrid system (wind and solar), this scenario representing to have wind turbine at buses 8,19 and 21 along with solar systems are

TABLE V. Solar only case

\begin{tabular}{|l|l|l|l|}
\hline & $P_{E S S}(\mathrm{MW})$ & $E_{E S S}(\mathrm{MWh})$ & Total cost(\$) \\
\hline Lead-acid & 293.9 & 947.6 & 514675.9 \\
\hline NiCd & 365.4 & 1601.1 & 510439.6 \\
\hline Li-ion & 393.4 & 1825.1 & 507687.8 \\
\hline NaS & 573.5 & 2568.8 & 500965.5 \\
\hline
\end{tabular}


TABLE VI. Hybrid case (Solar and wind)

\begin{tabular}{|l|l|l|l|}
\hline & $P_{E S S}(\mathrm{MW})$ & $E_{E S S}(\mathrm{MWh})$ & Total cost(\$) \\
\hline Lead-acid & 290.3 & 1179 & 403956.6 \\
\hline NiCd & 289 & 1247.7 & 403027.7 \\
\hline Li-ion & 250 & 957.1 & 403309.7 \\
\hline NaS & 378.9 & 1929.7 & 397531.1 \\
\hline
\end{tabular}

at buses 3,10 , and 14. from table it shows the output power and energy for each battery storage, the results illustrated all battery types are having close cost values. However the Nas ESS are an expensive solution. Comparing the hybrid system results with no renewable integration, the cost of the system for its best case is decreased from $\$ 0.532$ to $\$ 0.397$ which could save almost $26 \%$, in all cases the Nas shown to be the least expensive option.

\section{CONCLUSION}

In this paper, 24-hour horizon planning framework has been proposed for optimal allocation of DS units and sizing for distribution system considering different scenarios (wind, solar, hybrid and no renewable), to get the optimum operating cost by solving AC optimal power flow. The microgrid proposed is a 24- bus system, integrated with renewable resources and ESS. The study illustrates how the optimal selection of storage system could reduce the operating and ESS total cost. Integration of ESS in the network primarily provides more reliability and sustainability which makes better economical decision. Accordingly, a probabilistic approach is used to optimize the DS operation at each load state for achieving the arbitrage benefits. Furthermore, four different cases are discussed in this paper and results prove that integrating the DS units with distribution systems have the potential to reduce the overall cost, nevertheless, with control methodologies that can optimally deploy and utilize the energy resources. However, this is very promising in future which could be the optimal option when the investment cost of storage system become less to improve the system reliability and to rectify the power quality problems.

\section{ACKNOWLEDGMENT}

The authors would like to acknowledge the support provided by the Deanship of Research (DSR) at King Fahd University of Petroleum Minerals (KFUPM) for funding this work through project No. RG171009. Also, we would like acknowledge the funding support by the King Abdullah City for Atomic and Renewable Energy (K.A.CARE).

\section{REFERENCES}

[1] A. S. Awad, T. H. El-Fouly, and M. M. Salama, "Optimal ESS allocation for load management application," IEEE Transactions on Power Systems, vol. 30, no. 1, pp. 327-336, 2014.

[2] C. Chen, S. Duan, T. Cai, B. Liu, and G. Hu, "Optimal allocation and economic analysis of energy storage system in microgrids," IEEE Transactions on Power Electronics, vol. 26, no. 10, pp. 2762-2773, 2011.

[3] R. B. Schainker, "Executive overview: Energy storage options for a sustainable energy future," in IEEE Power Engineering Society General Meeting, 2004. Ieee, 2004, pp. 2309-2314.
[4] Y. M. Atwa and E. El-Saadany, "Optimal allocation of ess in distribution systems with a high penetration of wind energy," IEEE Transactions on Power Systems, vol. 25, no. 4, pp. 1815-1822, 2010.

[5] S. Jung and G. Jang, "A study on the voltage calculation method for ess operation plan in hybrid generation system Proc. of the Int. Conf. on Renewable Energies and Power Quality (ICREPQ'15), La Coruña, Spain."

[6] H. Bludszuweit and J. Domínguez, "Probabilistic energy storage sizing for reducing wind power forecast uncertainty," in International Conference on renewable energies and power quality (ICREPQ'10), Granada, Spain, 2010.

[7] F. A. Chacra, P. Bastard, G. Fleury, and R. Clavreul, "Impact of energy storage costs on economical performance in a distribution substation," IEEE Transactions on Power Systems, vol. 20, no. 2, pp. 684-691, 2005.

[8] C. Abbey and G. Joós, "A stochastic optimization approach to rating of energy storage systems in wind-diesel isolated grids," IEEE Transactions on Power Systems, vol. 24, no. 1, pp. 418-426, 2008.

[9] H. Lan, H. Yin, S. Wen, Y.-Y. Hong, D. Yu, and L. Zhang, "Electrical energy forecasting and optimal allocation of ESS in a hybrid wind-diesel power system," Applied Sciences, vol. 7, no. 2, p. 155, 2017.

[10] J. P. Barton and D. G. Infield, "A probabilistic method for calculating the usefulness of a store with finite energy capacity for smoothing electricity generation from wind and solar power," Journal of Power Sources, vol. 162, no. 2, pp. 943-948, 2006.

[11] G. Litjens, E. Worrell, and W. van Sark, "Assessment of forecasting methods on performance of photovoltaic-battery systems," Applied Energy, vol. 221, pp. 358-373, 2018.

[12] T. M. Masaud, O. Oyebanjo, and P. Sen, "Sizing of large-scale battery storage for off-grid wind power plant considering a flexible wind supplydemand balance," IET Renewable Power Generation, vol. 11, no. 13, pp. 1625-1632, 2017.

[13] A. Saez-de Ibarra, A. Milo, H. Gaztanaga, V. Debusschere, and S. Bacha, "Co-optimization of storage system sizing and control strategy for intelligent photovoltaic power plants market integration," IEEE Transactions on Sustainable Energy, vol. 7, no. 4, pp. 1749-1761, 2016.

[14] T. T. Tran and A. D. Smith, "Stochastic optimization for integration of renewable energy technologies in district energy systems for costeffective use," Energies, vol. 12, no. 3, p. 533, 2019.

[15] D. Simopoulos, Y. Giannakopoulos, S. Kavatza, and C. Vournas, "Effect of emission constraints on short-term unit commitment," in MELECON 2006-2006 IEEE Mediterranean Electrotechnical Conference. IEEE, 2006, pp. 973-977.

[16] C. Grigg, P. Wong, P. Albrecht, R. Allan, M. Bhavaraju, R. Billinton, Q. Chen, C. Fong, S. Haddad, S. Kuruganty et al., "The ieee reliability test system-1996. a report prepared by the reliability test system task force of the application of probability methods subcommittee," IEEE Transactions on Power Systems, vol. 14, no. 3, pp. 1010-1020, 1999.

[17] I. Alsaidan, A. Khodaei, and W. Gao, "A comprehensive battery energy storage optimal sizing model for microgrid applications," IEEE Transactions on Power Systems, vol. 33, no. 4, pp. 3968-3980, 2017. 